\title{
EXERCISE-INDUCED CHANGES IN URINARY WATER AND MINERAL OUPUT DURING THE MENSTRUAL CYCLE
}

\author{
D. N. PRASHAD, BSc, PhD, Senior Lecturer in Physiology, P. A. FLETCHER, BSc, PhD, Senior Lecturer in Physiology and \\ M. COOPER, BSc, PhD, Principal Lecturer in Physiology
}

\begin{abstract}
This study monitors changes in urinary excretion of water and minerals before and after exercise in pre-menopausal women and in those using oral contraception. Resting urine flow rate was lower and sodium output significantly higher ( $p<0.02$ ) during the normal post-menstrual phase than during other phases of the cycle. These changes were unrelated to glomerular filtration rate and might impede maximal performance. After exercise an oliguria and hyponatriuresis were most pronounced during the menstrual phase. In addition the resting urinary output of calcium and magnesium were unrelated to normal oestrogenic and progestegenic cyclic surges.
\end{abstract}

Amongst oral contraceptive users, resting excretion of water, minerals and creatinine were all significantly lower than general menstrual cycle values $(p<0.01)$ and further reductions in salts and water output occurred with exercise. Basal calcium and magnesium excretion rates were significantly lower than general menstrual cycle values $(p<0.05)$. Renal conservation of water and salts for 'pill' takers may affect renal homeostasis. The consequences of mineral retention are unknown.

Key words: Kidney function, Exercise, Contraceptive pill, Menstrual cycle, Water excretion, Mineral excretion.

\section{INTRODUCTION}

A physiololgical appraisal of the changes in body function during exercise, particularly with respect to respiratory and cardiovascular adjustments has been well documented (Mathews and Fox, 1976). Comparatively few studies have been undertaken to evaluate the important role of the renal system during exercise and yet exercise-renal interaction is of great significance to the athlete. The limited studies that have investigated this interaction generally have used male subjects, for example Zambraski et al (1974). Studies of exercise-renal interaction in females are rare.

It is now generally agreed that female physiological responses to exercise of a given intensity are quantitatively but not qualitatively different from those of males. Whether or not physical performance in females is significantly affected by menstruation appears to depend more upon the individual (Zaharieva, 1965). Nonetheless, fluctuations in ovarian hormone levels over the menstrual cycle may alter renal water and mineral output. It is possible that for some female athletes, these alterations may be sufficient to diminish peak performance efficiency.

It has been demonstrated previously that progesterone competes with aldosterone for its renal receptors (Fanstil and Park, 1981) and since progesterone levels increase during the pre-menstrual phase of the cycle this could result in a hypernatriuresis leading to extracellular depletion of sodium ions. In addition, elevated oestrogens occurring at ovulation and at the pre-menstrual phase of the cycle may act to conserve water and also synergistically with parathyroid hormone (Pitkins, 1976) to alter profoundly the renal output of minerals and water during these stages of the cycle.

The present study evaluates the normal and postexercise output of water and minerals during the menstrual, post-menstrual and pre-menstrual phases of the cycle of amateur female athletes. In addition, consideration is given to the effect that ultra-low dose oral contraceptives may have upon these physiological parameters.

Address for correspondence:

School of Biological Sciences and Environmental Health

Thames Polytechnic

Wellington Street

London

SE18 6PF

\section{MATERIALS AND METHODS}

Thirty female physical education students of approximately the same age $(20.4 \pm 0.7 \mathrm{yr})$ weight $(63.0 \pm 1.8 \mathrm{~kg})$, and height $(163.2 \pm 1.3 \mathrm{~cm})$ participated in this programme. Volunteers were divided into two groups, the first group comprising of those women who were menstruating regularly. In this group ( $n=16$ ) each participant monitored her menstrual cycle and the cycle was deemed to be regular if 21 to 36 days long. Each cycle was then standardised to 28 days and subdivided into three phases: the menstrual phase which corresponds to low plasma levels of both progesterone and oestrogen; the post-menstrual phase indicative of low progesterone and high oestrogen levels and the pre-menstrual phase corresponding to high levels of both steroids (Strott et al, 1970; Godfrey, 1981).

The second group $(n=14)$ of volunteers was made up of those women taking an ultra-low dose combined pill on a twenty-one-day regimen consisting either of $30 \mu \mathrm{g}$ ethinyloestradiol and $0.15 \mathrm{mg}$ levenorgesterol or $20 \mu \mathrm{g}$ ethinyloestradiol and $1 \mathrm{mg}$ norethisterone acetate. No significant difference was found between the two groups with regard to age or weight.

Following the selection of the two groups, timed urine samples were obtained from each participant one hour prior to exercise. The exercise routine consisted of running on a "Power Jog" treadmill for eighteen minutes with increasing speeds from 0 to $18 \mathrm{~km} \cdot \mathrm{hr}^{-1}$ at increments of $3 \mathrm{~km} \cdot \mathrm{hr}^{-1}$ every three minutes for 12 minutes and with the last 6 minutes run at $18 \mathrm{~km} \cdot \mathrm{hr}^{-1}$. However, although subjects' age, fitness and body weights were similar, it must be acknowledged that this routine did not represent the same relative exercise intensity for all subjects. At the end of the exercise protocol, timed urine samples were obtained from each subject.

The measurements described above were made during each of the three phases of this cycle in normallymenstruating women and on one occasion only, between the tenth and seventeenth day of the cycle, in the group taking oral contraceptives.

An aliquot of each urine sample was then stored at $-20^{\circ} \mathrm{C}$ for subsequent analysis. Urinary creatinine and sodium chloride ions were estimated using standard spectrophotometric and flame photometric methods respectively (Varley et al, 1980 and Wootton, 1964). Urinary 
calcium and magnesium were assayed by using an "SP191" atomic absorption spectrophotometer.

\section{RESULTS}

\section{Urine flow and Creatinine output}

Fig. 1(a) shows the mean basal (resting) urine flow over the three phases of the menstrual cycle. The post-menstrual water excretion was found to be lower by about $15 \%$ and $20 \%$ respectively compared with the menstrual and premenstrual phases of the cycle. This may be attributed to the oestrogenic surge occurring at the post-menstrual phase of the normal cycle.

Following exercise urinary output decreased significantly, by $35 \%$, ( $p<0.001$ ) during all three stages of the cycle with a more pronounced decline during the menstrual phase. Among contraceptive pill users (o/c users), basal urine output was about $40 \%$ lower than during each of the three periods of the normal menstrual cycle ( $p<0.01$ ), but declined further by only about $30 \%$, subsequent to exercise.

Fig. 1(b) shows the basal urinary excretion of creatinine over the three phases of the cycle. No significant interphasic changes were noted (Fig. 1(b)). Subsequent to exercise creatinine excretion reduced by approximately the same amount in each phase. The basal urinary excretion of creatinine for o/c users was significantly lower than those values obtained during the normal cycle ( $p<0.002$ ). Postexercise output of creatinine amongst this group was found to be reduced by only $10 \%$ (Fig. 1(b)).

Using the Pearsons correlation coefficient analysis a significant correlation was obtained when water output and creatinine excretion were compared prior to exercise for the normal group; ( $n=146 ; p<0.005 ; r=0.237$ ). However, following exercise no significant correlation was observed for these parameters. Amongst o.c. users no correlation was found before and/or after exercise. a

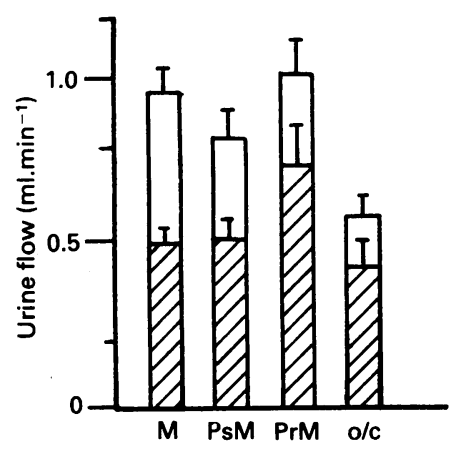

b

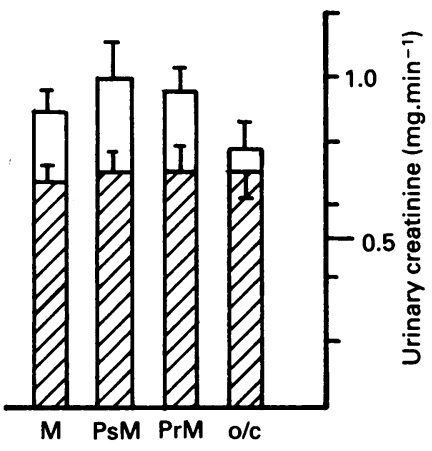

Fig. 1: Urine flow and urinary creatinine excretion (mean \pm SEM) before exercise $(\square ; n=54$ ) and after exercise ( $\square ; n=30)$, during menstruation (M), post-menstruation (PSM) and pre-menstruation (PrM), and for contraceptive pill users $(0 / c)$.

\section{Urinary excretion of sodium and chloride ions}

Fig. 2 shows that the basal urinary excretion of sodium was significantly higher during the post-menstrual period than at other phases of the cycle $(p<0.02)$. No marked changes in urinary chloride were observed prior to exercise. However, subsequent to exercise, the output of both sodium and chloride ions decreased significantly ( $p<0.001$ ) by about $50 \%$ and $35 \%$ respectively relative to normal resting levels, irrespective of the cycle.
Amongst the o/c users there was a significant retention of both sodium and chloride ions even before exercise shown by approximately $35 \%$ decline in output for each of these constituents relative to normal. The post-exercise urinary output of these constituents showed further reductions of almost $30 \%$ for sodium and $50 \%$ for chloride.

Based on the Pearson correlation coefficient, analysis of the data for normal subjects prior to exercise showed that sodium and chloride excretion were significantly correlated ( $p<0.01 ; n=149 ; r=0.235$ ). Furthermore, amongst the o/c users group basal sodium and chloride output were also found to be correlated $(r=0.45 ; n=23 ; P<0.001)$ but subsequent to exercise no such relationship was observed. a

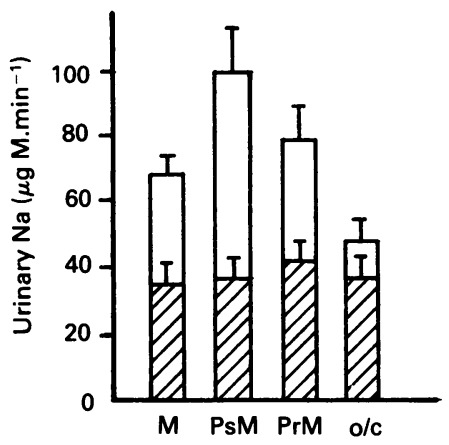

b

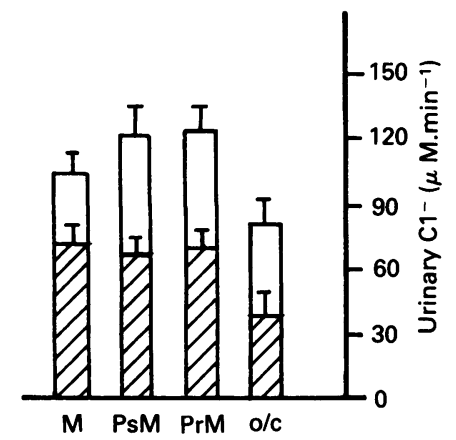

Fig. 2: Urinary Sodium and Chloride excretion (mean \pm SEM) before exercise $(\square ; n=54)$ and after exercise $(\square ; n=30)$, during menstruation $(M)$, postmenstruation (PsM) and pre-menstruation (PrM), and for contraceptive pill users $(o / c)$.

\section{Urinary excretion of Calcium and Magnesium}

The basal urinary excretion of calcium showed some fluctuations over the three phases of the cycle. However, subsequent to exercise significant reductions in urinary calcium output were found at all three phases of the cycle ( $p<0.001 ; p<0.02 ; p<0.01$ respectively). Amongst the o/c users basal calcium excretion rate was about $50 \%$ lower than during all phases of the cycle ( $p<0.001$ ) and postexercise calcium output declined even further $(50 \%$ reduction) (Fig. 3a).

The renal excretion of magnesium prior to exercise showed a progressive decline, from the menstrual to the pre-menstrual phase (Fig. 3b). Post-exercise excretion rates of magnesium were significantly lower when compared with individual phases of the cycle $(p<0.001 ; p<0.001$ and $p<0.05)$. As with calcium ouput, the excretion rate of magnesium amongst the o/c users prior to exercise was significantly lower compared with that observed over the three phases of the normal cycle declining further by about $40 \%$ after exercise.

\section{DISCUSSION}

The present investigation has demonstrated that the oliguria associated with moderate exercise occurred at all three phases of the menstrual cycle despite the oestrogenic and progestegenic surges occurring during the cycle. This decline in urine flow may be attributed to the previously observed fall in renal blood flow associated with physical exertion in which blood is presumed to be shunted away from splanchnic areas to exercising muscle (Eiser and Swartz, 1979; and accounting for a $20 \%$ reduction in cardiac output to the kidneys. Amongst the contraceptive pill users the resting urinary output of water was found to be 
significantly lower than for the non-o/c users and it is tempting to suggest that this reduction may also result from a similar redistribution of blood. However, no work has been found that will support this suggestion. Subsequent to exercise a further $25 \%$ reduction was noted for this o/c pill group compared with a significant $35 \%$ general reduction over the menstrual cycle.

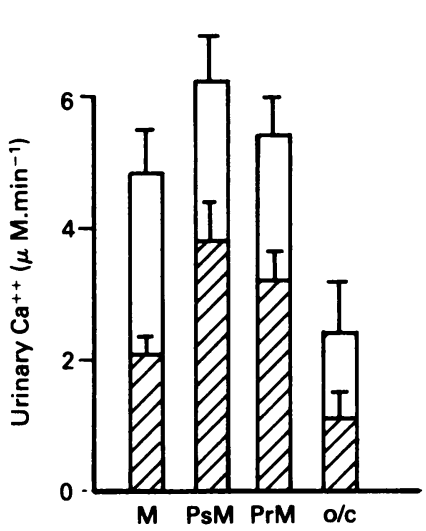

b

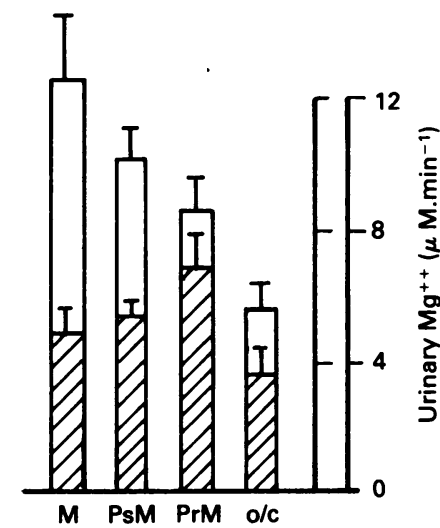

Fig. 3: Urinary Calcium and Magnesium excretion (mean \pm SEM) before exercise $(\square ;=54)$ and after exercise ( $\square ; n=30)$, during menstruation (M), post-menstruation (PsM) and pre-menstruation (PrM), and for contraceptive pill users $(0 / c)$

There is some evidence that peak performance levels decline during the pre- and post-menstrual phase of the normal cycle (Erdelyi, 1962) and in this study the lowest basal output of water was observed at the post-menstrual phase of the cycle. The massive retention of water shown in the current work to occur during this phase in normallymenstruating women may well be a factor contributing to this diminished quality of performance. Likewise, the low urinary flow noted amongst contraceptive pill users prior to and subsequent to exercise may act adversely with regard to performance.

The basal urinary excretion of creatinine showed no interphasic changes suggesting no steroidal influence on the urinary output of creatinine. The renal clearance of creatinine has been used previously as a crude measure of glomerular filtration rate (Pitts, 1974) and in the current study, after exercise the rate of urinary creatinine output declined. Assuming a normal blood creatinine level of $0.01 \mathrm{mg} \cdot \mathrm{ml}^{-1}$ and a post-exercise rate of creatinine output of $0.8 \mathrm{mg} \cdot \mathrm{min}^{-1}$ the renal clearance of creatinine was found to be $70 \mathrm{ml} \cdot \mathrm{min}^{-1}$ compared with normal values for women of $110 \mathrm{ml} \cdot \mathrm{min}^{-1}$. It is probable that such a decline in filtration rate may be related to a $50 \%$ reduction in renal blood flow observed previously (Eiser \& Swartz, 1979) in the exercising subject. Furthermore, since urine flow and creatinine output subsequent to exercise were found to be significantly correlated ( $p<0.005$ Fig. 1 ) it is possible that the output of both constituents resulted, at least in part, from a reduction in filtration rate. This decline in filtration rate could have resulted from renal arteriolar vasoconstriction effected by the increased renin activity known to occur during exercise (Collier et al, 1975).

The urinary excretion of sodium prior to exercise was found to be significantly higher $(p<0.002)$ at the postmenstrual phase than at the pre-menstrual phase coinciding with the high oestrogen/progesterone ratio known to occur at the former phase. Subsequent to exercise renal salt output declined significantly at all phases of the cycle. This massive reduction in urinary salt output may be related to arteriolar pressor effects of angiotension II, and/or a hyper-secretion of aldosterone. However, the situation may be more complicated since high levels of progesterone are known to antagonise the renal effects of aldosterone (Fanestil and Park, 1981). Whether, such antagonism occurred is not clear from the present studies since no marked differences were observed in renal salt retention at the luteal phase before and after exercise compared with those observed at the follicular and ovulatory stages of the cycle.

The basal urinary salt excretion amongst the oral contraceptive pill users was significantly lower than that observed during the normal cycle; and declined further after exercise. The mechanisms by which the reduced salt output is effected in this group before and after exercise are not fully understood. Nevertheless, it is suggested that the exogenous steroids may be exerting their effect on glomerular haemodynamics to reduce filtration rate before and during exercise. An increased secretion of renin induced by oestrogens may have contributed to the hyponatriuresis observed.

Urinary sodium output may be influenced by a hypersecretion of parathormone but in the context of this investigation changes in parathormone levels were not ascertained, and to the authors' knowledge no direct experimental evidence exists to show that parathormone levels increased during exercise. However, in this study an increased hydroxy-prolinuria (unpublished observations), an index of bone collagen turnover, was observed subsequent to exercise amounting to a $50 \%$ increase from resting levels for both groups under investigation. The basal urinary excretion of calcium declined subsequent to exercise at all phases of the cycle. Furthermore, amongst the oral contraceptive users group a significant reduction in urinary calcium was noted before exercise when compared with output observed over the cycle. These changes in calcium output may also be related to changes in renal haemo-dynamics which could affect the magnitude of all constituents currently investigated.

In general these findings indicate that the oral contraceptive pill may be influencing alteration in glomerular activity or to a lesser extent tubular activity and since the output of creatinine decreased, it is tempting to suggest that one of the major influences of the contraceptive pill on the kidney may be its direct or indirect effects on glomerular haemo-dynamics. Such an influence could alter the renal handling of a variety of blood filtrands appearing in the urine. However, in a simultaneous study (unpublished) it has been shown that after the 21 day pill taking regimen the massive renal conservation of the filtrands studied even before exercise was reversed within 3 days to basal levels comparable with those found over the normal menstrual cycle. Whether such a reversal can and does occur, over the long term, and whether the renal conservation mediated by the pill can lead to changes in renal homeostasis with/without augmented mineral deposition in calcified and soft tissues has not as yet been established.

\section{References}

Collier, J. G., Keddie, J. and Robinson, B. T., 1975 "Plasma activity during and after dynamic or status exercise". Cardiovasc.Res. 9: 323-328.

Eizer, A. R. and Swartz, 1979 "Renal and electrolyte alterations during exercise". In therapeutics through exercise edited by Grunel and Stratton inc., 51st Hahnemann Symposium, 145-153. 
Erdelyi, G., 1962 "Gynaecological survey of female athletes". J.Sports Med. 2: 174-179.

Fanstil, D. D. and Park, C. S., 1981 "Steroid hormones and the kidney". Ann.Rev.Physiol. 43: 637-649.

Fox, E. L. and Mathews, D. K., 1981 "Cardiorespiratory considerations, Section $3^{\prime \prime}$. In The physiological basis of physical education and athletics. Saunders College pubs. 3rd edition, 183-251.

Pitkin, R. M., Reynolds, W. A., Williams, G. A. and Hargis, G. K., 1978 "Calcium regulating hormones during the menstrual cycle". J.Clin.Endocrin.Metab. 47: 626-632.

Pitts, R. F., 1972 "Physiology of the kidney and body fluids". Yr.bk.Med.Pubs. 3rd edition, 60-95.
Strott, C. A., Yoshimi, T., Ross, C. T. and Lipsett, M. B., 1969 "Ovarian physiology relationship between plasma $\mathrm{LH}$ and steroidogenesis by the follicle and corpus luteum; effect of HCG". J.Clin.Endocr. 29: 1157-1167.

Varley, H., Gowenlock, A. H. and Bell, M., 1976. Practical Clinical Biochemistry. Vol. 2, 5th edition, Heinemann, London.

Wootton, I-D. P., 1964 Micro-analysis in medical biochemistry. J. A. Churchill (Ed.).

Zaharieva, S., 1965 "Survey of sports women at the Tokyo Olympics". J.Sports Med.Phys. Fitness 5: 215-217.

Zambraski, E. J., Tipton, C. M., Jordan, H. R., Palmer, W. K. and Tcheng, T. K. 1974 "lowa wrestling study; urinary profiles of state finalists prior to competition". Med. \& Sci. in sports 6: 129-132.

Title:

BOOK REVIEW

Author:

\title{
RUNNING WITHOUT FEAR
}

Publisher:

Kenneth H. Cooper

Bantam Books

Price: $£ 3.95$

\author{
ISBN 055317356
}

This is the latest in a series of books on exercise written by Kenneth Cooper, the US doctor often credited with coining the term 'aerobics'. The title presupposes that people are afraid whilst running and requires explanation. The book was written to allay the anxiety raised in the minds of Americans when Jim Fixx, committed jogger and author of the best-selling book "The Complete Book of Running", died whilst out running. This event re-opened the debate about the relative risks and advantages of taking exercise and "Running without Fear" is Cooper's contribution to this debate. His preoccupation with the individual case of Jim Fixx is evident throughout the book. The first 70 pages (of 209) are devoted to the topic of sudden death during exercise but there are also chapters on understanding coronary risk factors, on suitable forms of aerobic exercise and on exercise stress tests (with much emphasis on the protocol used in the Cooper Clinic).

It is difficult to know for what audience the book is intended. The analysis of coronary risk factors is a useful and readily understood summary for the layman who may also appreciate the practical advice about topics such as warm up. He is unlikely, however, to have much interest in or understanding of details of abnormal ECG responses to a treadmill stress test and subsequent diagnosis and management (Chapter 8). On the other hand, the clinician would doubtless prefer to share Cooper's undoubted wealth of experience with exercise testing through the scientific literature. No reference is made to such publications in the list of selected recent papers on risks and benefits of exercise which follows the text. The book is a curious mixture of anecdotal, even evangelical, tales about the role of exercise in maintaining health and a serious attempt to describe and interpret the scientific evidence of this topic.

Adrianne E. Hardman

\section{BOOK REVIEW}

Title:

\section{ESSENTIALS OF SPORTS MEDICINE}

Author:

Greg R. McLatchie

Publisher: $\quad$ Churchill Livingstone, Edinburgh

Price: $f 14.95 \quad$ ISBN 0443030448

The name of Greg McLatchie is sufficient in itself to be a guarantee for this excellent introduction to Sports Medicine. The immediate appeal of this compilation is that it introduces subjects rarely found in other books on the subject. There are contributions on sport and the law, the role of exercise in prevention and treatment of disease and a code of ethics for doctors involved in treating sports players. These are all subjects of considerable concern, particularly when there is increasing public interest in the medical problems of the famous and increasing public participation in sport. In a book of only 200 pages or so, covering the whole range of Sports Medicine from diet to sudden death, from training to treatment, it is not surprising that subjects are not covered in depth. It is a book on the essentials of Sports Medicine and the essentials it deals with very well. I particularly liked the section on physiotherapy and strapping, subjects that most of us know little about.

One or two minor niggles. Chondromalacia patella has largely been abandoned as a diagnosis and I would venture to suggest that the physical signs described for anterior knee pain are not all that common. Incidentally, the main description of the condition is not indexed. As may be expected, there is an emphasis on injuries in combat and contact sports, perhaps at the expense of the more popular running and racquet sports. Stress fractures, which are an increasingly common problem, receive little coverage and again the indexing,js suspect, one reference being to another page of the index. However, all in all, a good book for all GPs prepared to take an interest in their patients' sporting life and certainly useful reading for those embarking on the BASM courses. Available, on loan, from the BASM library. 